\title{
Real-World Experience of Endoscopic Submucosal Dissection for Ulcerative Colitis-Associated Neoplasia
}

\author{
Akira Matsui Shu Hoteya Junnosuke Hayasaka Satoshi Yamashita \\ Yorinari Ochiai Yugo Suzuki Yumiko Fukuma Takayuki Okamura \\ Yutaka Mitsunaga Masami Tanaka Kousuke Nomura Nobuhiro Dan \\ Hiroyuki Odagiri Daisuke Kikuchi
}

Department of Gastroenterology, Toranomon Hospital, Tokyo, Japan

\section{Keywords \\ Ulcerative colitis-associated neoplasia - Endoscopic submucosal dissection - Negative horizontal margin rate}

\begin{abstract}
Introduction: Patients with ulcerative colitis (UC) have an increased risk of colorectal cancer. Some studies have recently investigated endoscopic resection of UC-associated neoplasia (UCAN), but the indications for endoscopic resection of UCAN remain controversial. This study sought to clarify the problems encountered in endoscopic submucosal dissection (ESD) for UCAN. Methods: Seventeen lesions in $12 \mathrm{pa}-$ tients with UCAN (UCAN group) and 913 epithelial lesions in 824 control patients without UC (non-UC group) were evaluated. Both groups underwent ESD between January 2010 and December 2017 at Toranomon Hospital, Tokyo, Japan. Treatment outcomes of the 2 groups were compared retrospectively. Results: Univariate analysis showed that the mean tumor size was significantly smaller in the UCAN group than in the non-UC group $(25.1 \pm 26.7 \mathrm{~mm}$ vs. $31.9 \pm 19.0$;
\end{abstract}

karger@karger.com www.karger.com/iid

Karger $\stackrel{\text { ' }}{5}$

GOPEN ACCESS
(C) 2021 The Author(s)

Published by S. Karger AG, Basel

This is an Open Access article licensed under the Creative Commons Attribution-NonCommercial-4.0 International License (CC BY-NC) (http://www.karger.com/Services/OpenAccessLicense), applicable to the online version of the article only. Usage and distribution for commercial purposes requires written permission. $p=0.0023)$; however, the R0 resection rate was significantly lower in the UCAN group (70.6 vs. $92.9 \% ; p=0.001$ ). Multivariate analysis showed a significantly lower negative horizontal margin rate in the UCAN group (odds ratio 11.3, 95\% confidence interval 3.588-34.525; $p=0.000$ ). Discussion/ Conclusion: ESD for UCAN is associated with a low-negative horizontal margin rate. When performing ESD for UCAN, it is important to evaluate the accuracy of the UCAN demarcation line, especially for flat lesions, using white-light imaging and chromoendoscopy as well as other modalities, including biopsy of surrounding tissues.

(C) 2021 The Author(s)

Published by S. Karger AG, Basel

\section{Introduction}

The risks of dysplasia and cancer are high in patients with ulcerative colitis (UC) [1-3]. The standard treatment for UC-associated neoplasia (UCAN) is total colectomy because the characteristics of UCAN are unclear and endoscopic diagnosis is difficult [4]. Recently, the 
rates of endoscopic diagnosis of dysplasia and intramucosal cancer have been improved by high-resolution endoscopy and chromoendoscopy [5]. The SCENIC (Surveillance for Colorectal Endoscopic Neoplasia Detection and Management in Inflammatory Bowel Disease Patients: International Consensus Recommendations) guidelines [6] have classified macroscopic types of dysplasia found by endoscopy into polypoid, nonpolypoid, and invisible. According to the European Crohn's and Colitis Organization guidelines [7], endoscopic resection of polypoid dysplasia is acceptable, but there is no evidence for endoscopic resection of lesions with nonpolypoid dysplasia.

The outcomes of endoscopic resection for UCAN have been reported. Iacopini et al. [8] reported that the R0 resection rate was $80 \%$ for nonpolypoid UCAN following ESD. No recurrence was seen during a median observation period of 24 months, even though margin delineation was difficult in 5 cases and submucosal fibrosis was observed in 9 cases. Kinoshita et al. [9] reported an R0 resection rate of $76 \%$ and a negative lateral margin rate of $100 \%$, but all lesions that were not R0 resected had a positive vertical margin. In this study, we aimed to clarify the problems encountered in ESD in patients with UCAN by comparing their outcomes with those of patients with epithelial tumors but without UC.

\section{Materials and Methods}

\section{Patients and Lesions}

Seventeen lesions of 12 patients with UCAN (UCAN group) and 913 epithelial lesions in 824 patients without UC (non-UC group) were retrospectively compared. Both groups underwent ESD at the Department of Gastroenterology, Toranomon Hospital, Tokyo, Japan, between January 2010 and December 2017. This study was approved by the Institutional Review Board of Toranomon Hospital. Written informed consent for surgery was obtained from all patients prior to the ESD.

The indication for ESD in both groups was an estimated depth of submucosal invasion $<1,000 \mu \mathrm{m}$ measured on chromoendoscopy magnifying endoscopy or endoscopic ultrasonography. Endoscopically invisible UCAN lesions with an unclear border and lesions in patients with endoscopically moderate-to-severe active UC were excluded. All lesions were observed by white-light endoscopy and chromoendoscopy with indigo carmine as well as magnifying narrowband imaging and crystal violet staining before ESD. The demarcation line of the lesion was identified by chromoendoscopy with indigo carmine dye, magnification, and pathological diagnosis using biopsies from the surrounding mucosa near the lesion. The macroscopic type of each lesion was defined based on the SCENIC guidelines [6].
Endoscopic Submucosal Dissection Technique for UC-

Associated Neoplasia

ESD was performed using the dual knife (Olympus, Tokyo, Japan) through a PCF-Q260JI/GIF-Q260J endoscope with a water-jet function (Olympus) as described previously [10]. In brief, a soft transparent Olympus hood was attached to the tip of the endoscope to obtain direct endoscopic views of the submucosal layer. Marking dots were placed on the normal mucosa approximately $5 \mathrm{~mm}$ from the tumor margin to provide safety margins for UCAN. After submucosal injection of a $10 \%$ glycerol $/ 5 \%$ fructose solution (Chugai Pharmaceutical, Tokyo, Japan) containing $0.005 \%$ indigo carmine and $0.0005 \%$ epinephrine, a mucosal incision was made outside the marking dots. Hyaluronic acid solution was added to the injection solution when mucosal elevation was insufficient due to ulceration of the lesion or extensive fibrosis of the submucosal layer. After mucosal incision, the submucosal layer was dissected directly to obtain an intact specimen and allow complete en bloc resection. Hemostatic forceps (Olympus) were used to control bleeding during the procedure. ESD was usually performed under conscious sedation using pethidine hydrochloride $(35-70 \mathrm{mg})$.

Postoperative bleeding was defined as bloody stool requiring an endoscopic hemostatic procedure after ESD. Perforation during the procedure was sutured by clipping. After procedure, we confirmed by detection of free air on a plain radiograph. Endoscopic balloon dilatation was performed when the endoscope could not pass a stenosis due to an ESD ulcer scar.

All ESD procedures were performed by an endoscopist who had sufficient experience to safely perform ESD for upper digestive tumors independently. The endoscopist who performed ESD in the patients with UCAN had previously performed $>100$ colorectal ESD procedures unrelated to UC. Nine endoscopists performed ESD in non-UC group, and 5 out of 9 endoscopists performed ESD in UCAN group.

\section{Definitions and Evaluation}

UCAN was defined as follows: (1) a definitive diagnosis of UC, (2) inflammation from UC in the mucosa around the tumor or past inflammation on endoscopy, and (3) histological diagnosis of UCAN confirmed by routine hematoxylin and eosin staining and p53 immunochemistry. We evaluated for overexpression or complete absence of $\mathrm{p} 53$, basal positivity for $\mathrm{p} 53$, and the presence of UC-associated inflammation in the surrounding lesions. We also defined UCAN when Ki-67 was expressed in all layers. Sporadic adenomas were defined as (1) tumors that formed outside the area affected by UC or (2) polypoid or superficially elevated tumors that formed within the area affected by UC and showed spotty or focal p53 staining. Tumors found within 1 year of the date of the first ESD were defined as synchronous, and those found more than 1 year after the date of the first ESD were defined as metachronous.

We reviewed the characteristics of the patients with UCAN and analyzed their short- and long-term outcomes following ESD. Univariate and multivariate analyses were performed to compare the following parameters between the 2 groups: sex, age, tumor size, histological type, depth of tumor invasion, negative lymphovascular invasion rate, negative horizontal and vertical margin rates, submucosal fibrosis rate, $\mathrm{R} 0$ resection rate, and complications. The primary outcome was the $\mathrm{R} 0$ resection rate. Secondary outcomes were tumor size, depth of tumor invasion, lymphovascular invasion rate, negative horizontal and vertical margin rates, 
Table 1. Clinical characteristics of patients with UC

\begin{tabular}{lc}
\hline Characteristic & Value, $n=12$ \\
\hline Sex & \\
$\quad$ Male/female, $n(\%)$ & $6(50): 6(50)$ \\
Age, mean \pm SD, years & $59.3 \pm 14.5$ \\
Duration of disease, mean \pm SD, years & $20.4 \pm 10.8$ \\
Extent, $n(\%)$ & \\
$\quad$ Extensive & $10(83.3)$ \\
$\quad$ Left-sided & $2(16.7)$ \\
$\quad$ Proctitis & $0(0)$ \\
Medication for UC, $n(\%)$ & $1(8.3)$ \\
$\quad$ None & $7(58.3)$ \\
$\quad$ Mesalazine & $2(16.7)$ \\
$\quad$ PSL & $2(16.7)$ \\
IFX & $1.33 \pm 1.82$ \\
Mayo score, mean \pm SD, points & $0.58 \pm 0.51$ \\
Mayo endoscopic subscore, mean \pm SD, points & \\
\hline
\end{tabular}

IFX, infliximab; PSL, prednisolone; SD, standard deviation; UC, ulcerative colitis.

and complications. R0 resection was defined as en bloc resection with horizontal and vertical margins that were free of histopathologic evidence of tumor.

\section{Statistical Analysis}

Data are presented as means \pm standard deviations. The statistical analysis was carried out using the $\chi^{2}$ test, Wilcoxon rank-sum test, and logistic regression analysis. All statistical analyses were performed using Intercooled Stata ${ }^{\mathrm{TM}} 11.0$ for Windows (Stata Corp., College Station, TX, USA). A $p$ value $<0.05$ was considered statistically significant.

\section{Results}

\section{Characteristics of Patients with UCAN}

Patients in the UCAN group had a mean age of $59.3 \pm$ 14.5 years and a mean disease duration of $20.4 \pm 10.8$ years; $83.3 \%$ had extensive disease (Table 1 ). The mean Mayo score during ESD was $1.33 \pm 1.82$ and the mean Mayo endoscopic subscore was $0.58 \pm 0.51$, indicating clinically mild disease activity to remission. Many patients showed mucosal healing.

\section{Clinicopathological Findings and Outcomes in the \\ UCAN Group}

UCAN was localized to the rectum in $58.8 \%$ of cases, the macroscopic type was the superficial elevated type in $58.8 \%$ of cases according to the SCENIC classification, and the nonpolypoid type was common (Table 2). The mean tumor diameter was $25.1 \pm 26.7 \mathrm{~mm}$; $35.3 \%$ of the
Table 2. Clinicopathological findings in patients with UCAN

\begin{tabular}{|c|c|}
\hline Characteristic & Value, $n=17$ \\
\hline \multicolumn{2}{|l|}{ Location, $n(\%)$} \\
\hline Rectum & $10(58.8)$ \\
\hline Sigmoid & $3(17.6)$ \\
\hline Descending & $2(11.8)$ \\
\hline Ascending & $2(11.8)$ \\
\hline \multicolumn{2}{|l|}{ Macroscopic type, $n(\%)$} \\
\hline Polypoid sessile & $1(5.9)$ \\
\hline Superficial elevated & $10(58.8)$ \\
\hline Flat & $6(35.3)$ \\
\hline Tumor size, mean $\pm \mathrm{SD}, \mathrm{mm}$ & $25.1 \pm 26.7$ \\
\hline \multicolumn{2}{|l|}{ Major histologic type, $n(\%)$} \\
\hline Tubular adenoma & $2(11.8)$ \\
\hline Low-grade dysplasia & $4(23.5)$ \\
\hline High-grade dysplasia & $4(23.5)$ \\
\hline Differentiated adenocarcinoma & $7(41.2)$ \\
\hline \multicolumn{2}{|l|}{ Histologic type, $n(\%)$} \\
\hline Monomorphic & $11(64.7)$ \\
\hline Mixed & $6(35.3)$ \\
\hline \multicolumn{2}{|l|}{ Depth of tumor invasion, $n(\%)$} \\
\hline Intramucosal & $17(100)$ \\
\hline Submucosal & $0(0)$ \\
\hline \multicolumn{2}{|l|}{ Lymphovascular invasion, $n(\%)$} \\
\hline Negative & $17(100)$ \\
\hline Positive & $0(0)$ \\
\hline \multicolumn{2}{|l|}{ Horizontal margin, $n(\%)$} \\
\hline Negative & $12(70.6)$ \\
\hline Unevaluable & $3(17.6)$ \\
\hline Positive & $2(11.8)$ \\
\hline \multicolumn{2}{|l|}{ Vertical margin, $n(\%)$} \\
\hline Negative & $17(100)$ \\
\hline Positive & $0(0)$ \\
\hline
\end{tabular}

SD, standard deviation; UCAN, ulcerative colitis-associated neoplasia.

lesions were a mixture of histological types, and the depth of tumor invasion was mucosal in all cases. Notably, the negative horizontal margin rate was low $(70.6 \%)$. The R0 resection rate was also $70.6 \%$.

Two of the 17 lesions (11.8\%) were stenosed after ESD; all lesions were treated with circumferential ESD (Table 3). One of the cases with stenosis after ESD was a whitish, superficial elevated lesion extending from $\mathrm{Rb}$ to Ra (Fig. 1). In this case, the anal side of the lesion was in contact with the squamous epithelium of the anus and was seen circumferentially (Fig. 1a, b). The oral side of the lesion had spread to Ra for a maximum of two-thirds (Fig. 1c, d). Marking dots were placed with reference to the results of the peripheral biopsy done before ESD (Fig. 1e). ESD was performed circumferentially on the anal side and involved threequarters of the circumference on the oral side (Fig. 1f). A 
Table 3. Outcomes of ESD in patients with UCAN

\begin{tabular}{llc}
\hline Outcome & Value (12 patients, 17 lesions) \\
\hline Observation period, months mean \pm SD (range) & & $28.5 \pm 23.2(5-82)$ \\
Observation rate, $n(\%)$ & $11 / 12$ patients & 91.7 \\
Follow-up, months mean \pm SD (range) & & $24.7 \pm 23.6(4-82)$ \\
Total colectomy rate after ESD, $n(\%)$ & $1 / 12$ patients & 8.3 \\
R0 resection rate, $n(\%)$ & $12 / 17$ lesions & 70.6 \\
Complications, $n(\%)$ & Delayed bleeding 0/17 lesions & 0 \\
& Perforation 0/17 lesions & 0 \\
Local recurrence rate, $n(\%)$ & Stenosis $2 / 17$ lesions & 11.8 \\
Synchronous tumor rate, $n(\%)$ & $0 / 17$ lesions & 0 \\
Metachronous tumor rate, $n(\%)$ & $3 / 12$ patients & 25.0 \\
Overall survival rate, $n(\%)$ & $1 / 12$ patients & 8.3 \\
\end{tabular}

"Observation" means at least 1 visit after ESD or patients for whom outcomes were confirmed. "Follow-up" is the time interval from the date of ESD to the last surveillance colonoscopy. ESD, endoscopic submucosal dissection; SD, standard deviation; UCAN, ulcerative colitis-associated neoplasia.

total of 6 endoscopic balloon dilations were performed from 69 to 198 days after ESD (Fig. 1g). In another case, ESD was performed for a lesion with unclear margins in the sigmoid colon. Because the horizontal margin was positive pathologically, an additional ESD procedure was performed 10 days later. This patient underwent full-circumference ESD that included the first ESD ulcer. In total, 3 endoscopic balloon dilatations were performed in the 2746 days after the second ESD. During a mean observation period of 28.5 months, the local recurrence rate was $0 \%$, the synchronous tumor rate was $25.0 \%$, and the metachronous tumor rate was $8.3 \%$.

\section{Horizontal Margin-Negative Lesions versus}

\section{Horizontal Margin-Positive Lesions}

In this study, the horizontal margin was positive in 2 cases and unevaluable in 3 cases. One of the 3 unevaluable cases underwent circumferential ESD of the rectum, so the horizontal margin could not be evaluated. In 1 case, thermal degeneration occurred on the anal side of the tumor, precluding evaluation of the horizontal margin. In the remaining unevaluable case and the 2 positive cases, the demarcation line of dysplasia around the superficial elevated lesion could not be detected.

We compared the endoscopic features of the lesions according to whether their horizontal margins were negative or positive. In conventional white-light images, dendritic blood vessels were clearly seen around an elevated lesion (Fig. 2a, c) and could be considered an endoscopic feature of a negative horizontal margin. However, in the
2 cases with a positive horizontal margin, plastic mucosa with poor color change was extensively observed, and no dendritic blood vessels were evident (Fig. 3a, c). On chromoendoscopy with indigo carmine, the mucosal pattern around the elevated lesion was relatively uniform in the cases with a negative horizontal margin (Fig. 2b, d); however, the pattern was uneven in the positive cases, and it was difficult to judge whether the change was inflammatory or neoplastic (Fig. 3b, d). The characteristic endoscopic findings for identifying the demarcation line were not clear on magnifying endoscopy with narrowband imaging and crystal violet spraying.

\section{Comparison of Patient Characteristics in the UCAN and Non-UC Groups}

Univariate analysis revealed that the UCAN group, compared with non-UC group, had significantly lower mean age $(59.25 \pm 14.5$ years vs. $66.8 \pm 10.7$ years; $p=$ $0.0173)$, mean tumor size $(25.1 \pm 26.7 \mathrm{~mm}$ vs. $31.9 \pm 19.0$ $\mathrm{m} ; p=0.0023$ ), negative horizontal margin rate (70.6 vs. $94.7 \% ; p=0.000)$, and R0 resection rate (70.6 vs. $92.9 \%$; $p=0.001$; Table 4). Furthermore, the UCAN group had a significantly longer mean procedure time $(155 \pm 133 \mathrm{~min}$ vs. $110 \pm 74 \mathrm{~min} ; p=0.0315$ ). There was no significant difference between the groups in specimen size; however, the specimen/tumor size ratio was significantly larger in the UCAN group ( $3.33 \pm 3.13$ vs. $1.44 \pm 1.10 ; p=0.0000)$. The stenosis rate was significantly higher in the UCAN group (11.8 vs. $0.66 \% ; p=0.000)$, but there was no significant difference in the delayed bleeding rate or perforation rate. 


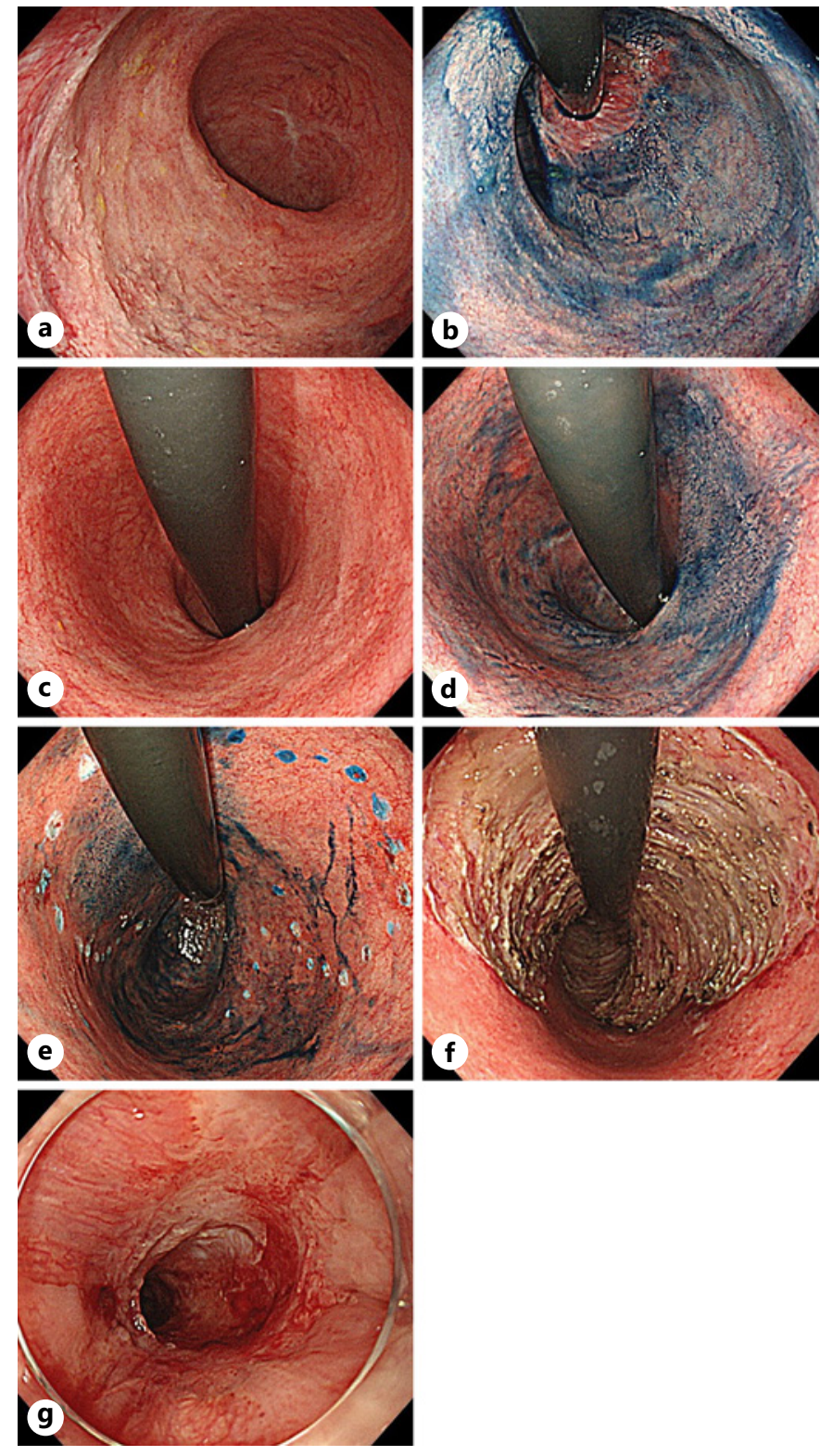

Fig. 1. Endoscopic images of a case of stenosis. a White-light image of the anal side of the lesion. $\mathbf{b}$ Image of the anal side of the lesion obtained by chromoendoscopy with indigo carmine. c White-light image of the oral side of the lesion. $\mathbf{d}$ Image of the oral side of the lesion obtained by chromoendoscopy with indigo carmine. e Image of the lesion with marking dots obtained by chromoendoscopy with indigo carmine. $\mathbf{f}$ White-light image of an ESD ulcer. $\mathbf{g}$ Whitelight image of the stenosis 69 days after ESD. ESD, endoscopic mucosal dissection.

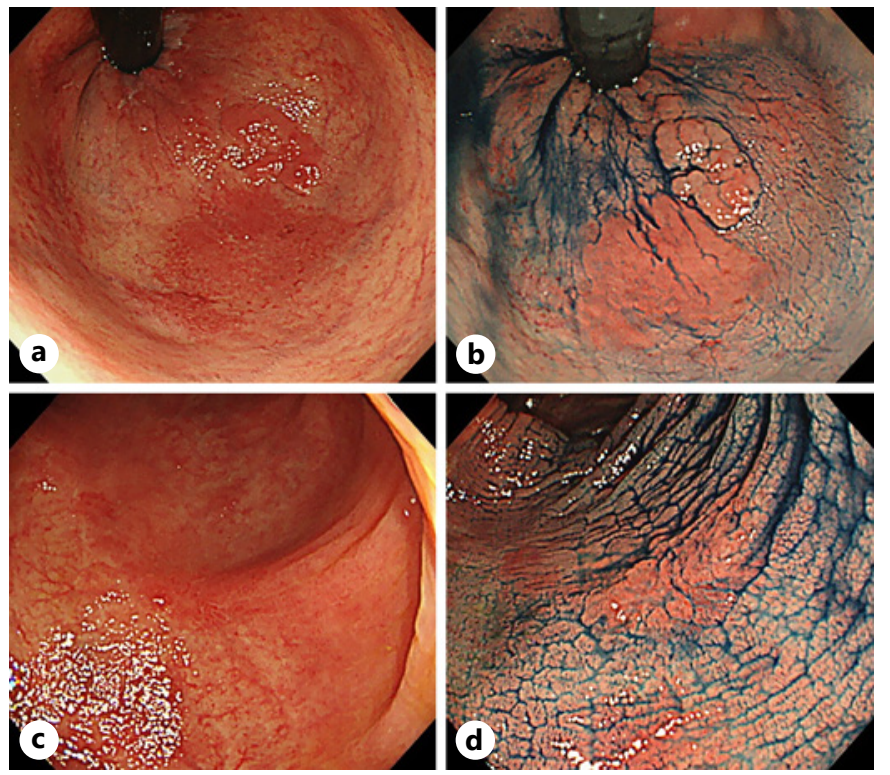

Fig. 2. Endoscopic images of cases with a negative horizontal margin. a White-light image of a lesion with a negative horizontal margin obtained from case 1 . b An image of the same lesion in case 1 obtained by chromoendoscopy with indigo carmine. c White-light image of a lesion with a negative horizontal margin obtained from case 2. $\mathbf{d}$ An image of the same lesion in case 2 obtained by chromoendoscopy with indigo carmine.

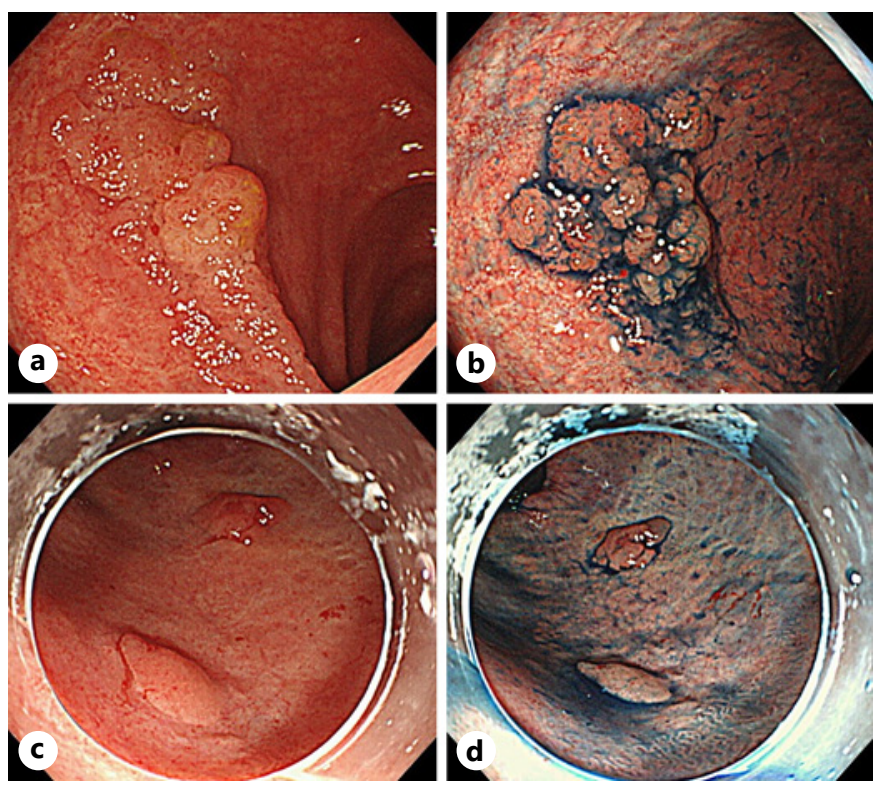

Fig. 3. Endoscopic images from cases with a positive horizontal margin and cases that could not be evaluated. a White-light image of a lesion with a positive horizontal margin. $\mathbf{b}$ An image of the same lesion obtained by chromoendoscopy with indigo carmine. c White-light image of a lesion in which the horizontal margin could not be evaluated. $\mathbf{d}$ An image of the same lesion obtained by chromoendoscopy with indigo carmine. 
Table 4. Univariate analysis of the tumor after ESD in the UCAN and non-UC groups

\begin{tabular}{lccc}
\hline Characteristic & $\begin{array}{l}\text { UCAN group, } \\
n=17\end{array}$ & $\begin{array}{l}\text { Non-UC group, } \\
n=913\end{array}$ & $p$ value \\
\hline Age, mean \pm SD, years & $59.25 \pm 14.5$ & $66.8 \pm 10.7$ & 0.0173 \\
Male sex, $n$ (\%) & $8(47.1)$ & $534(58.5)$ & 0.344 \\
Tumor size, mean \pm SD, mm & $25.1 \pm 26.7$ & $31.9 \pm 19.0$ & 0.0023 \\
Specimen size, mean \pm SD, mm & $46.1 \pm 27.9$ & $40.2 \pm 18.6$ & 0.6866 \\
Specimen/tumor size ratio, mean \pm SD & $3.33 \pm 3.13$ & $1.44 \pm 1.10$ & 0.0000 \\
Procedure time, mean \pm SD, min & $155 \pm 133$ & $110 \pm 74$ & 0.0315 \\
Histological type, mixed, $n(\%)$ & $8(47.1)$ & $492(53.9)$ & 0.576 \\
Depth of invasion, intramucosal, $n(\%)$ & $17(100)$ & $757(82.9)$ & 0.175 \\
Lymphatic invasion negative, $n(\%)$ & $17(100)$ & $879(96.3)$ & 0.720 \\
Vascular invasion negative, $n(\%)$ & $17(100)$ & $875(95.8)$ & 0.692 \\
Horizontal margin negative, $n(\%)$ & $12(70.6)$ & $865(94.7)$ & 0.000 \\
Vertical margin negative, $n(\%)$ & $17(100)$ & $893(97.8)$ & 0.537 \\
Submucosal fibrosis positive, $n(\%)$ & $1(5.9)$ & $129(14.1)$ & 0.331 \\
R0 resection, $n(\%)$ & $12(70.6)$ & $848(92.9)$ & 0.001 \\
\hline Complications, $n(\%)$ & & & 0.517 \\
Delayed bleeding & $0(0)$ & $22(2.4)$ & 0.664 \\
Perforation & $0(0)$ & $10(1.1)$ & 0.000 \\
Stenosis & $2(11.8)$ & $6(0.66)$ & \\
\hline
\end{tabular}

UC, ulcerative colitis; UCAN, ulcerative colitis-associated neoplasia; ESD, endoscopic submucosal dissection.

Table 5. Multivariate analysis

\begin{tabular}{lllll}
\hline & OR & SE & $p$ value & 95\% CI \\
\hline Tumor size & 0.9659766 & 0.0184927 & 0.071 & $0.9304032-1.00291$ \\
Specimen/tumor size ratio & 1.212812 & 0.1014413 & 0.021 & $1.029432-1.428858$ \\
Procedure time & 1.006174 & 0.0019655 & 0.002 & $1.002329-1.010033$ \\
Submucosal fibrosis & 0.2213803 & 0.2372302 & 0.159 & $0.0271016-1.808351$ \\
Horizontal margin & 11.09294 & 6.749357 & 0.000 & $3.366232-36.55521$ \\
\hline
\end{tabular}

CI, confidence interval; OR, odds ratio; SE, standard error.

Multivariate analysis showed that the UCAN group had significantly lower negative horizontal margin rate (odds ratio [OR] 11.09, 95\% confidence interval [CI] $3.366-36.555 ; p=0.000)$ and significantly longer operating time (OR 1.006, 95\% CI 1.002-1.010; $p=0.002$; Table 5). In addition, the UCAN group had a significantly greater specimen/tumor size ratio (OR 1.213, 95\% CI $1.029-1.429 ; p=0.021$ ).

\section{Discussion/Conclusion}

To our knowledge, this is the first study to compare the efficacy and safety of ESD between patients with UCAN and control patients with epithelial lesions not associated with $\mathrm{UC}$. In this study, the $\mathrm{R} 0$ resection rate was significantly lower in the UCAN group (70.6\%) than in the non-UC group (92.9\%), which could reflect the significantly lower negative horizontal margin rate in the UCAN group found in multivariate analysis. The negative horizontal margin rate was $70.6 \%$ in this study, which is considered to be due to inflammatory or postinflammatory changes in the mucosa around the UCAN lesion. Even though the average Mayo endoscopic subscore in the patients with UCAN was only $0.58 \pm 0.51$, it was difficult in some cases to make a clear endoscopic diagnosis of the demarcation line between inflammatory mucosa with low-disease activity and dysplasia. Furthermore, diagnosis of the demarcation line may be difficult in UCAN when the dysplasia spreads horizon- 
tally. This raises the question of how to detect the demarcation line.

We consider peripheral biopsy before ESD to be important for detecting the demarcation line. Shinagawa et al. [11] reported that pine cone and villi patterns are endoscopic findings suggestive of UCAN. Moreover, Nishiyama et al. [12] recommend that the focus should be on high residual density of pits and regular pit margins observed under magnifying chromoendoscopy when differentiating between UCAN and nonneoplastic lesions. Therefore, attention should be paid to such findings when identifying the demarcation line. It has been reported that acetic acid should be used in combination with indigo carmine to detect the demarcation line when performing ESD for gastric tumors [13]. It has also been suggested that spraying sessile serrated adenomas/polyps with acetic acid and indigo carmine together is useful for borderline diagnosis [14]. However, no studies have investigated the use of acetic acid to detect the margin of UCAN. We did not examine this in our study given the lack of information on the safety of spraying acetic acid on the mucosa in patients with UC.

We believe that chromoendoscopy makes it easier to identify endoscopic features that help to detect the demarcation line, especially when indigo carmine is used. By spreading indigo carmine around the tumor and surrounding tissue, taking care to avoid foaming and having a distant view, it is possible to identify the spread of lesions and demarcation lines that cannot be seen on whitelight imaging. Furthermore, we recommend that after identifying an adequate demarcation line endoscopically, biopsies be obtained from the surrounding tissues and be pathologically confirmed before performing ESD. However, given that biopsy scars can make ESD difficult, we do not recommend tumor biopsy in patients without UC; nevertheless, biopsy scars were not a major factor complicating ESD in our UCAN group. Furthermore, there was no significant difference in the frequency of submucosal fibrosis between the 2 groups in this study. These observations raise the question of why the procedure time was significantly longer in the UCAN group. The reason is that peripheral biopsy and marking of dots are needed before ESD, which increases the size of the resected specimen. Therefore, in this study, we examined the size of the resected specimen relative to the size of the tumor and found that this ratio was significantly larger in the UCAN group, which indicates that the procedure time would be long even if the tumor diameter were small. The high stenosis rate was probably the result of increasing the resection area in order to achieve a negative horizontal margin.
Although this study has some limitations, including a single-center, retrospective observational design and a small sample size, we consider that its findings have clinical relevance. The low rates of local recurrence and total colectomy after ESD suggest that total colectomy can be avoided in some cases of UCAN by performing ESD.

Detection of the demarcation line is more difficult when ESD is performed for UCAN than when it is performed for lesions not associated with UC. Adequate consideration of the demarcation line is essential when performing ESD for UCAN.

\section{Acknowledgement}

The authors thank the clinical staff of the Department of Gastroenterology and Pathology at our institution for the excellent care they provided during this study.

\section{Statement of Ethics}

This study was approved by the Institutional Review Board of Toranomon Hospital and was conducted in accordance with the Declaration of Helsinki (1964 and its later versions). Written informed consent for inclusion in the study was not required in view of the retrospective observational nature of the research and the anonymity of the data. However, patient consent was obtained using the opt-out method via our website.

\section{Conflict of Interest Statement}

The authors have no conflicts of interest to declare.

\section{Funding Sources}

The authors did not receive any funding.

\section{Author Contributions}

A.M. and S.H. designed the study. A.M. wrote the initial draft of the manuscript in consultation with S.H. and D.K. All authors contributed to the analysis and interpretation of the data. J.H. and S.Y. contributed to data collection and interpretation with Y.O., T.O., Y.S., Y.F., N.D., Y.M., M.T., K.N., and H.O. All authors critically reviewed the manuscript, approved its final version, and agree to be held accountable for all aspects of the work by ensuring that questions related to the accuracy or integrity of any part of the work are appropriately investigated and resolved.
Matsui et al. 


\section{References}

1 Ekbom A, Helmick C, Zack M, Adami HO. Ulcerative colitis and colorectal cancer. A population-based study. N Engl J Med. 1990 Nov;323(18):1228-33.

2 Eaden JA, Abrams KR, Mayberry JF. The risk of colorectal cancer in ulcerative colitis: a meta-analysis. Gut. 2001 Apr;48(4):526-35.

3 Hata K, Watanabe T, Kazama S, Suzuki K, Shinozaki M, Yokoyama T, et al. Earlier surveillance colonoscopy programme improves survival in patients with ulcerative colitis associated colorectal cancer: results of a 23-year surveillance programme in the Japanese population. Br J Cancer. 2003 Oct;89(7):1232-6.

4 Itzkowitz SH, Harpaz N. Diagnosis and management of dysplasia in patients with inflammatory Bowel diseases. Gastroenterology. 2004 May;126(6):1634-48.

5 Picco MF, Pasha S, Leighton JA, Bruining D, Loftus EV Jr, Thomas CS, et al. Procedure time and the determination of polypoid abnormalities with experience: implementation of a chromoendoscopy program for surveillance colonoscopy for ulcerative colitis. Inflamm Bowel Dis. 2013 Aug;19(9):1913-20.
6 Laine L, Kaltenbach T, Barkun A, McQuaid KR, Subramanian V, Soetikno R. SCENIC international consensus statement on surveillance and management of dysplasia in inflammatory bowel disease. Gastrointest Endosc. 2015 Mar;81(3):489-e26.

7 Margo F, Gionchetti P, Eliakim R, Ardizzone $\mathrm{S}$, Armuzzi A, Barreiro-de Acosta M, et al. Third european evidence-based consensus on diagnosis and management of ulcerative colitis. Part1: definitions, diagnosis, extra-intestinal manifestations, pregnancy, cancer surveillance, surgery, and ileo-anal pouch disorders. J Crohns Colitis. 2017 Jun;11(6):648-70.

8 Iacopini F, Saito Y, Yamada M, Grossi C, Rigato $\mathrm{P}$, Costamagna G, et al. Curative endoscopic submucosal dissection of large nonpolypoid superficial neoplasms in ulcerative colitis (with videos). Gastrointest Endosc. 2015 Oct; $82(4): 734-8$.

9 Kinoshita S, Uraoka T, Nishizawa T, Naganuma M, Iwao Y, Ochiai Y, et al. The role of colorectal endoscopic submucosal dissection in patients with ulcerative colitis. Gastrointest Endosc. 2018 Apr;87(4):1079-84.
10 Nakajima T, Saito Y, Tanaka S, Iishi H, Kudo $\mathrm{SE}$, Ikematsu H, et al. Current status of endoscopic resection strategy for large, early colorectal neoplasia in Japan. Surg Endosc. 2013 Sep;27(9):3262-70.

11 Shinagawa T, Hata K, Morikawa T, Takiyama $\mathrm{H}$, Emoto S, Murono K, et al. Pine-cone and villi patterns are endoscopic signs suggestive of ulcerative colitis-associated colorectal cancer and dysplasia. Gastrointest Endosc. 2019 Mar;89(3):565-e3.

12 Nishiyama S, Oka S, Tanaka S, Hayashi N, Hayashi R, Nagai K, et al. Is it possible to discriminate between neoplastic and nonneoplastic lesions in ulcerative colitis by magnifying colonoscopy? Inflamm Bowel Dis. 2014 Mar;20(3):508-13.

13 Sakai Y, Eto R, Kasanuki J, Kondo F, Kato K, Arai M, et al. Chromoendoscopy with indigo carmine dye added to acetic acid in the diagnosis of gastric neoplasia: a prospective comparative study. Gastrointest Endosc. 2008 Oct;68(4):635-41.

14 Yamamoto S, Shafazand M. Acetic acid-indigocarmine mixture for evaluating the margins of sessile serrated adenomas/polyps. Dig Endosc. 2017 Nov;29(7):817-8. 